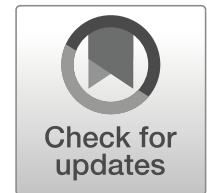

\title{
Outcomes of honeybee pupae inoculated with deformed wing virus genotypes $A$ and $B$
}

\author{
Eric Dubois, Marine Dardouri, Frank Schurr, Nicolas Cougoule, \\ Fabrice SiRCOULOMB, Richard ThIÉRY

\begin{abstract}
ANSES, Sophia Antipolis Laboratory, Unit of Honey Bee Pathology, 105 route des Chappes - CS20111, F-06902, Sophia Antipolis, France
\end{abstract}

Received 26 February 2019 - Revised 9 August 2019 - Accepted 9 October 2019

\begin{abstract}
Deformed wing virus and Varroa destructor virus-1 have a high percentage of nucleotide identity and might be considered as closely related viruses: DWV genotype A (DWV-A) and DWV genotype B (DWV-B) respectively. They have been implicated in overwinter colony losses in association with Varroa destructor infestations that vectored both DWV variants. In this study, we performed experimental inoculations of honeybee pupae with viral suspensions prepared from honeybee heads naturally infected by either DWV-A or DWV-B. Two outcomes were observed: the inoculated pupae exhibited either higher rates of bees with deformed wings or higher mortality rates than control bees. For both DWV variants, the viral loads quantified in the head of inoculated bees were significantly greater than those in control bees $(p<0.01)$. These outcomes were not correlated to the virus genotype (DWV-A or DWV-B) detected in the inocula by RT-quantitative PCR targeting the VP3 coding sequence (RT-qPCR). However, the highest mortality rates found in our study were correlated with an increase in sacbrood virus (SBV) load. Despite the fact that only either DWV-A or DWV-B was expected to be inoculated, we observed increased mortality in honeybees that were infected with both DWV and SBV.
\end{abstract}

\section{Deformed wing virus / Varroa destructor virus-1 / virulence / wing deformity / pupae mortality}

\section{INTRODUCTION}

Deformed wing virus (DWV) and Varroa destructor virus-1 (VDV-1) are two related viruses (family Iflaviridae; genus Iflavirus) belonging to the same viral cloud of DWV variants: DWV genotype A (DWV-A) and DWV genotype B (DWV-B) respectively (de Miranda et al. 2010; Lanzi et al. 2006; Ongus et al. 2004). Both variants have been detected in honeybee colonies on several continents (Martin et al. 2012; Mordecai et al. 2016a; Strauss et al. 2013). However, VDV1 appears to have spread more recently than DWV

Corresponding author: E. Dubois, eric.dubois@ anses.fr Manuscript Editor: Michelle L Flenniken around the world (Ongus et al. 2004; Ryabov et al. 2017). Recombinant viruses between both variants have also been characterized by sequencing (Cornman 2017; Dalmon et al. 2017; Moore et al. 2011; Ryabov et al. 2014; Zioni et al. 2011). Most of the recombinant genomic RNA showed similarities with a viral protein-coding sequence derived from VDV-1 and a non-structural proteincoding sequence derived from DWV. A third variant (DWV type C) has more recently been detected in Devon, England (Mordecai et al. 2016b) and high viral titers were quantified in collapsing colonies (Kevill et al. 2017). However, the prevalence and impact of this DWV variant in the rest of the world are unknown.

Not all but some DWV strains could have replicative activity in the Varroa destructor mite, which preferentially transmits them to honeybees 
(Bowen-Walker et al. 1999; Gisder et al. 2009; Gisder et al. 2018; Ongus et al. 2004; Yue and Genersch, 2005). Even when $V$. destructor infestation is well controlled by chemical treatment, the established DWV variants can still be transmitted horizontally to larvae by trophallaxis with infected nurse bees or by the consumption of contaminated pollen (Chen et al. 2006; Locke et al. 2017; Mondet et al. 2014; Yue and Genersch, 2005). Vertical transmission by the queen's unfertilized eggs or drone sperm has also been reported (Amiri et al. 2017; Yue et al. 2007). These transmission routes lead to covert infections with no obvious symptoms, and the viral loads quantified by RTquantitative PCR are frequently below $10^{7}$ equivalent viral genome copies per bee (Forsgren et al. 2012; Martin et al. 2012; Mondet et al. 2014). Horizontal transmission by the $V$. destructor mite is associated with a reduction in the DWV variant's diversity, and/or to the increase in viral loads producing overt diseases (Di Prisco et al. 2011; Martin et al. 2012; Mondet et al. 2014). Recent studies demonstrated that mutualistic symbiosis between the mite and DWV affects humoral and cellular immune responses by interfering with NF- $K B$ signaling and enhances the reproduction of the parasitic mite (Di Prisco et al. 2016; Nazzi et al. 2012). Sub-lethal effects on adult honeybees include learning deficits (Gisder et al. 2018; Iqbal and Mueller, 2007), a reduction in foraging performance (Benaets et al. 2017; Wells et al. 2016) and a shorter life span of winter honeybees (Dainat et al. 2012a). Honeybee's aggressive behaviors was also correlated with the replication of the Kakugo strain (DWV-A) in the brain of workers (Fujiyuki et al. 2004; Shah et al. 2009). DWV variants and $V$. destructor are considered together to be predictive markers of colony collapse and winter losses (Dainat et al. 2012b; Genersch et al. 2010). The adverse effects of DWV are currently reduced by controlling mite infestations (chemically or naturally by hygienic colonies), which also protects the colony against winter mortalities (Francis et al. 2013; Locke et al. 2017). Nevertheless, other stress factors can trigger wing deformities or mortality in the absence of V. destructor (Forsgren et al. 2012). Notably, studies have shown that DWV replication in honeybees is stimulated by co-exposure to pesticides affecting honeybee immunity (DeGrandiHoffman et al. 2013; Di Prisco et al. 2013).

The severity of DWV infections can be influenced by mite infestation and transmission and/or attributed to nucleotide or strain variation. Specifically, Martin et al. detected DWV-A in collapsing colonies after the mite spread to the Hawaiian Islands (Martin et al. 2012). Mordecai et al. suggested in their study that DWV-B could dominate DWV-A by a viral competition mechanism and the new viral equilibrium was associated with lower virulence (Mordecai et al. 2016a). However, McMahon et al. demonstrated that the emerging DWV-B was more virulent than the established DWV-A (McMahon et al. 2016). The finding of resistant colonies to high mite infestations and high viral loads was not only correlated to honeybee tolerance (Locke et al. 2014) but could also be explained by these less virulent viral strains. Moreover, several studies report an increase in the virulence of recombinant viruses between DWV-A and DWV-B, suggesting that such recombinants would be adapted to being vectored by the mite (Moore et al. 2011; Ryabov et al. 2014). The comparison of these results can be complicated by the multiplicity of models under study — such as larvae, pupae, adult honeybees or $V$. destructor - and the virulence criteria monitored, including replicative potential, wing deformities, or death. Overt DWV infection is characterized by wing deformities and/or high viral loads being detected in all the infected honeybee's body parts, especially in total RNA extracted from the head (Genersch et al. 2010; Mockel et al. 2011; Yue and Genersch, 2005). Such a correlation between deformities and viral load in the head (above $10^{7}$ equivalent genomic copies) was also found in naturally infected honeybees (Zioni et al. 2011).

In this study, we performed experimental inoculation of honeybee pupae with viral suspensions prepared from naturally infected adult honeybee heads in order to evaluate the virulence of DWV$\mathrm{A}$ and DWV-B according to three criteria: rate of mortality, rate of wing deformities, and viral load in the head of inoculated honeybees. Our results are also discussed in the light of two recent studies on DWV inoculation of pupae (Remnant et al. 2019; Tehel et al. 2019). Notably, our results 
support the previous conclusion that DWV variants induce no significant increase in pupae death compared to control pupae.

\section{MATERIAL AND METHODS}

\subsection{Honeybee colony selection and sampling of pupae}

In November 2017, three healthy colonies were selected for their low viral loads of acute bee paralysis virus (ABPV), black queen cell virus (BQCV), chronic bee paralysis virus (CBPV), sacbrood virus (SBV), DWV-A and DWV-B, measured from a pool of 10 adult honeybees by quantitative RT-PCR (RT-qPCR) (Schurr et al. 2017; Schurr et al. 2019). In order to reduce mite infestation, each colony was treated with Amitraz (Apivar, Véto-pharma) for 1 month before being moved into the winter apiary. The hives were set up in a building designed to maintain a temperature allowing honeybee queens to lay eggs (21 ${ }^{\circ} \mathrm{C}$ ). Moreover, access points through the wall allowed the honeybees to fly outside the winter apiary for cleansing purposes. The colonies were fed with water, $50 \%$ sucrose syrup, and irradiated pollen mixed with sucrose syrup for four months. Between February 2018 (about 1 month after the queens started laying eggs again) and April 2018 (end of the winter leading to the foraging of honeybees in their natural environment), sealed brood combs were used to collect white-eyed pupae with entomological forceps. The natural reduction in the $V$. destructor mite was monitored daily for each colony (Dietemann et al. 2013). Moreover, pupae collected for the experiments and their corresponding brood cells were carefully inspected to confirm the absence of $V$. destructor. Viral loads from a pool of 10 adult honeybees were quantified at the beginning and end of the experiments.

\subsection{Experimental design}

DWV-A or DWV-B viral suspensions were obtained from naturally infected honeybee heads. Individual honeybee heads were crushed in $500 \mu \mathrm{l}$ of $10 \mathrm{mM}$ phosphate buffer, $\mathrm{pH} 7$ (PB) and the suspensions were clarified by two centrifugations for $10 \mathrm{~min}$ at $8000 \times \mathrm{g}$. The resulting supernatant was filtered through a polytetrafluoroethylene (PTFE) syringe filter with a $0.2-\mu \mathrm{m}$ porosity (Fisherbrand, Fisher Scientific). DWV-A and DWV-B loads were measured by RT-qPCR to be adjusted in $\mathrm{PB}$ at $10^{4}$ equivalent genome copies per $2 \mu \mathrm{l}$ or at lower levels. These inocula $(2 \mu \mathrm{l})$ were injected into pupae according to the standard method (Crailsheim et al. 2013; Mockel et al. 2011). Briefly, the carefully extracted white-eyed pupae were placed in cellulose-lined cavities of 24-well microtiter plates and kept in an incubator (35 ${ }^{\circ} \mathrm{C}, 60-70 \%$ humidity). The inocula were injected ventrally through the intersegmental membrane between the third and fourth abdominal segment using a hollow microliter needle. Control pupae were mock-infected with $2 \mu \mathrm{l}$ PB or not manipulated at all (non-inoculated pupae). All the pupae were kept in an incubator $\left(35^{\circ} \mathrm{C}\right.$, $60-70 \%$ humidity) until the end of the pupal stage. Pupae fatally injured by coarse piercing or handling became noticeable within $12 \mathrm{~h}$ postinjection by melanization and death. These pupae were removed from the experiments. In order to allow the complete development of all the pupae in the plate, a small hole filled with a few microliters of sucrose syrup was drilled in the lid above each well for 1 to 2 days following the emergence of the first honeybee. The rates of pupae mortality and of emerging honeybees with obvious disease symptoms (deformed wings) were evaluated. The plates were subsequently stored at $-80^{\circ} \mathrm{C}$ for further molecular analysis.

\subsection{Sample processing}

DWV-A and DWV-B viral loads in RNA extracted from the honeybees' head was used as an additional molecular marker for overt DWV infection (Schurr et al. 2019). Bees were decapitated and each head was crushed in $500 \mu \mathrm{l}$ of $10 \mathrm{mM}$ phosphate buffer ( $\mathrm{pH} 7.0$ ) with three $3 \mathrm{~mm}$ tungsten beads using Mixer Mill MM 400 (Retsch) for $1 \mathrm{~min}$ at $30 \mathrm{~Hz}$. The homogenate was clarified by two successive centrifugations at $8000 \times \mathrm{g}$ for 10 min at $4{ }^{\circ} \mathrm{C}$. Viral RNAs were extracted from $140 \mu \mathrm{l}$ of clarified homogenate with QIAamp Viral RNA Mini Kit (Qiagen), according to the manufacturer's instructions (final recovery volume $60 \mu \mathrm{l}$ ). Complementary DNA synthesis was 
performed using random primers and SuperScript's SSRT II kit (Invitrogen) from $12.5 \mu$ of purified RNA (400 ng of total RNA; final volume of reverse transcription mix $20 \mu \mathrm{l}$ ), and both DWV-A and DWV-B loads were quantified (using $5 \mu$ of complementary DNA; qPCR final volume $25 \mu \mathrm{l}$ ) by two genotype-specific qPCR amplifying the VP3-coding sequences (Table I), according to previous report (Schurr et al. 2019).

In order to describe the complete viral status of honeybees, the viral loads of ABPV, BQCV, CBPV, and SBV were also quantified by RTqPCR methods (Table I).

Threshold cycles $(\mathrm{Ct})$ from our samples were compared with standard curves establishing a linear relation between $\mathrm{Ct}$ and plasmid loads in the range of $1.0 \times 10^{2}$ to $1.0 \times 10^{9}$ copies $/ 5 \mu$ (Schurr et al. 2019). The viral load in honeybees was expressed in a decimal logarithm $\left(\log _{10}\right)$ of equivalent copies of viral genome per head (copies/head) using the following equation: copies/bee $=68.6 \times$ copies $/$ PCR . This conversion factor was calculated based on the volume used at each step of the RT-qPCR method.

\subsection{Statistical analyses}

The $95 \%$ confidence intervals $(95 \% \mathrm{CI})$ for the mortality and wing deformity rates were estimated using a binomial exact distribution (Greiner and Gardner, 2000), and rates were compared by a chi-square test. The viral loads $\left(\log _{10}\right.$-transformed values) were compared using Student's $t$ test. Statistical analyses were performed using R software (Version 1.0.143-(C) 2009-2016 RStudio). Differences were considered significant at $p<0.01$.

\section{RESULTS}

\subsection{Health status of the colonies}

Table II reports the number of $V$. destructor and viral loads quantified from a pool of 10 honeybees that were sampled from the three colonies before being moved into the winter apiary, at the start and end of the experimental period (in November 2017, January 2018, and April 2018 respectively). During the experiments, the mite
$V$. destructor was not detected in the three colonies. All the colonies sampled in January were negative for the six main honeybee viruses. However, BQCV, DWV-A, DWV-B, and SBV were quantified from a pool of 10 foragers collected at the end of the experiment (in April 2018).

\subsection{Outcomes and viral loads in control honeybees}

The rates for bees with wing deformities or dead pupae were measured for control pupae (i.e., that were either not inoculated or inoculated with PB) (Figure 1). Among 23 non-inoculated pupae (one experimental trial), 1 died during pupation $(4 \%)$ and none of the honeybees emerged with wing deformities $(0 \%)$. Over the six experimental trials of pupae inoculated with PB $(n=$ 108 ), the rates of death during pupation lay between 12 and $37 \%$, and the rates of emerging bees with wing deformities lay between 12 and $26 \%$ (Figure 2). The mean pupae mortality rate was $23 \%$ (95\% CI [15-31]), and the mean rate of wing deformities was $20 \%$ (95\% CI [13-28]). Consequently, 57\% (95\%CI [47-66]) of bees emerged with normal wings at the end of the experiments in the PB-inoculated control group.

Both DWV-A and DWV-B loads were quantified by RT-qPCR from the heads of control honeybees (Figure 3 and Table III). The viral loads quantified in PB-inoculated bees were no different from those measured in non-inoculated bees $(p>$ $0.01)$. The viral loads measured in honeybee heads with different outcomes (i.e., dead pupae, emerged bees with deformed and normal wings) from a PB-inoculated control group were not significantly different $(p>0.01)$. The DWV-A and DWV-B loads were below $7.8 \times 10^{5}$ and below $4.8 \times 10^{7}$ copies/head, respectively.

\subsection{Viruses detected in the inocula}

The DWV-A inocula 1 and 2 and DWV-B inoculum 1, were prepared from honeybees with deformed wings, while DWV-A inoculum 3 and DWV-B inoculum 2 were prepared from the head of honeybees with normal wings. Both DWV-A and DWV-B were quantified in the RNA extracted from these viral suspensions by RT-qPCR 
E. Dubois et al.

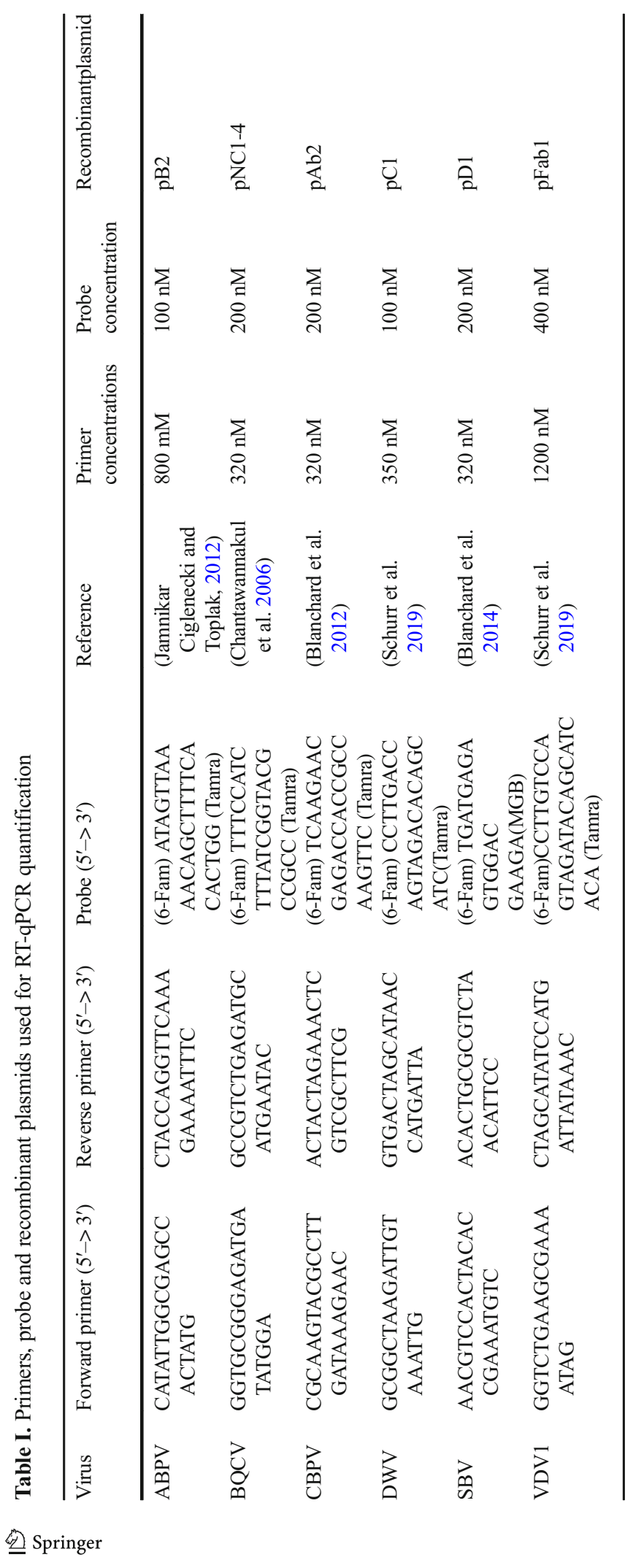


Table II. Health status of the honeybee colonies kept in the winter apiary

\begin{tabular}{|c|c|c|c|c|c|c|c|c|}
\hline \multirow{2}{*}{$\begin{array}{l}\text { Colony } \\
\text { number }\end{array}$} & \multirow[t]{2}{*}{ Sampling period ${ }^{1}$} & \multicolumn{6}{|c|}{ Viral loads (copies/bee) $)^{2}$} & \multirow{2}{*}{$\begin{array}{l}\text { Natural mite } \\
\text { fall for } 2 \\
\text { weeks } \\
\text { V. destructor }\end{array}$} \\
\hline & & ABPV & $\mathrm{BQCV}$ & CBPV & DWV-A & DWV-B & SBV & \\
\hline \multirow[t]{3}{*}{1} & November 2017 & $\mathrm{nd}^{3}$ & $2.2 \times 10^{4}$ & nd & nd & $1.6 \times 10^{4}$ & $7.7 \times 10^{4}$ & Amitraz $^{5}$ \\
\hline & January 2018 & nd & nd & nd & nd & nd & nd & 0 \\
\hline & April 2018 & nd & $6.7 \times 10^{4}$ & nd & nd & nd & $8.3 \times 10^{4}$ & 0 \\
\hline \multirow[t]{3}{*}{2} & November 2017 & nd & $1.8 \times 10^{4}$ & nd & nd & $1.4 \times 10^{4}$ & $7.7 \times 10^{4}$ & Amitraz \\
\hline & January 2018 & nd & nd & nd & nd & nd & nd & 0 \\
\hline & April 2018 & nd & $2.5 \times 10^{5}$ & nd & nd & $8.0 \times 10^{5}$ & $2.5 \times 10^{5}$ & 0 \\
\hline \multirow[t]{3}{*}{3} & November 2017 & nd & $1.0 \times 10^{3}$ & nd & $1.4 \times 10^{3}$ & $1.2 \times 10^{6}$ & $9.3 \times 10^{2}$ & Amitraz \\
\hline & January 2018 & nd & nd & nd & nd & nd & nd & 0 \\
\hline & April 2018 & nd & $1.7 \times 10^{9}$ & nd & nd & $3.2 \times 10^{6}$ & $9.5 \times 10^{5}$ & 0 \\
\hline
\end{tabular}

${ }^{1}$ Sampling dates: 14 November 2017 (Amitraz treatment of the colonies); 31 January 2018(start of the experiments), and 16 April 2018 (end of the experiments)

${ }^{2}$ The viral loads from a pool of 10 honeybees were quantified by quantitative RT-PCR

${ }^{3} n d$ not detected

${ }^{4}$ The number of naturally fallen mites was counted for the 2 weeks before the sampling period dates

${ }^{5}$ Colony treated with Amitraz for one month

(Table IV). DWV-A was the main virus detected in the three viral suspensions used to prepared DWV-A inocula 1 to 3, while DWV-B was the main virus detected in the remaining two used to prepared DWV-B inocula 1 and 2. Taking into account the dilution factors used to adjust the inocula, we calculated the contamination levels by other viruses. SBV was quantified at very low levels in all the inocula (SBV loads between 0.13 and 280 copies/2 $\mu$ l), while BQCV was quantified only in the viral suspension used to prepare the DWV-A inocula 1 to 3. (BQCV loads between 0.025 and 240 copies $/ 2 \mu \mathrm{l})$. ABPV and CBPV were not detected in the viral suspensions.

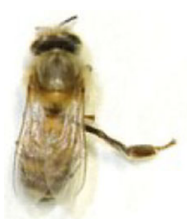

a

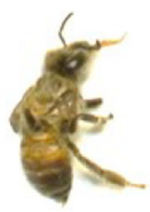

b

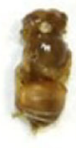

C
Figure 1. Symptoms observed during the emergence period in control bees inoculated with phosphate buffer. a Normal bee. b Bee with wing deformities. c Dead pupa.

\subsection{Outcomes of honeybees inoculated with $10^{4}$ equivalent genome copies of DWV variants}

The suspensions made from a single honeybee head were adjusted to $10^{4}$ equivalent of DWV variant genome copies per $2 \mu \mathrm{l}$ and inoculated into pupae. Two outcomes were observed (Figure 4). The injection of DWV-A inocula 1 and 3 or DWV-B inoculum 2 led to an increase in mortality rate $(>90 \%)$. All the pupae inoculated with DWV-A inoculum $3(n=11)$ or DWV-B inoculum $2(n=43)$ died within the 7 to 8 days of pupation. Among 15 pupae inoculated with DWV-A inoculum 1, 1 emerging bee had no deformities. The pupae inoculated with DWV-A inoculum $2(n=54)$ or DWV-B inoculum $1(n=$ $30)$ emerged with deformed wings (74-95\% CI [62-86] and 67-95\%CI [50-84] respectively) and few pupae mortalities were observed (2295\% CI [11-33] and 20-95\% CI [6-34] respectively). These mortality rates were not significantly different from that for control pupae inoculated with $\mathrm{PB}$ ( $p=0.68$ and $p=0.56$ respectively). However, the rates of wing deformities for 
a Pupae mortalities

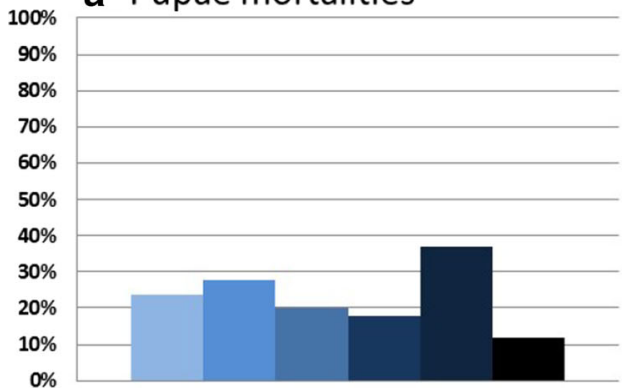

b Emerging bees with deformities

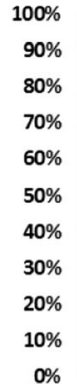

Figure 2. Rates of pupae mortality (a) and of emerging bees with wing deformities (b) in control bees by inoculating pupae with phosphate buffer. Between 17 and 20 pupae were experimented on during each trial.

emerging bees inoculated with DWV-A inoculum 2 or DWV-B inoculum 1 were significantly higher than that for PB control bees (both $p<0.01$ ). No correlation was found between the genotypes of the inoculated DWV variant and the rate of wing deformities or the rate of pupae mortalities. Both inocula containing DWV-A (DWV-A inoculum 2) or DWV-B (DWV-B inoculum 1) were associated with emerging bees with deformed wings and low mortality rates. In addition, both DWV-A (DWVA inocula 1 and 3) and DWV-B (DWV-B inoculum 2) were associated with high rates of pupae mortality.

\subsection{Viral loads in the head of honeybees inoculated with $10^{4}$ equivalent genome copies of DWV variants}

The loads of DWV variants (genotype A or B) were quantified by RT-qPCR in the heads of honeybees from the experimental inoculations with $10^{4}$ copies/pupae (Figure 5). Surprisingly, pupae inoculated with inocula containing DWV-A (DWV-A inocula 1 to 3) or DWV-B (DWV-B inocula 1 and 2) showed an increase in both DWV-A and DWV-B loads at the end of experimentation. For each experiment, the viral loads of

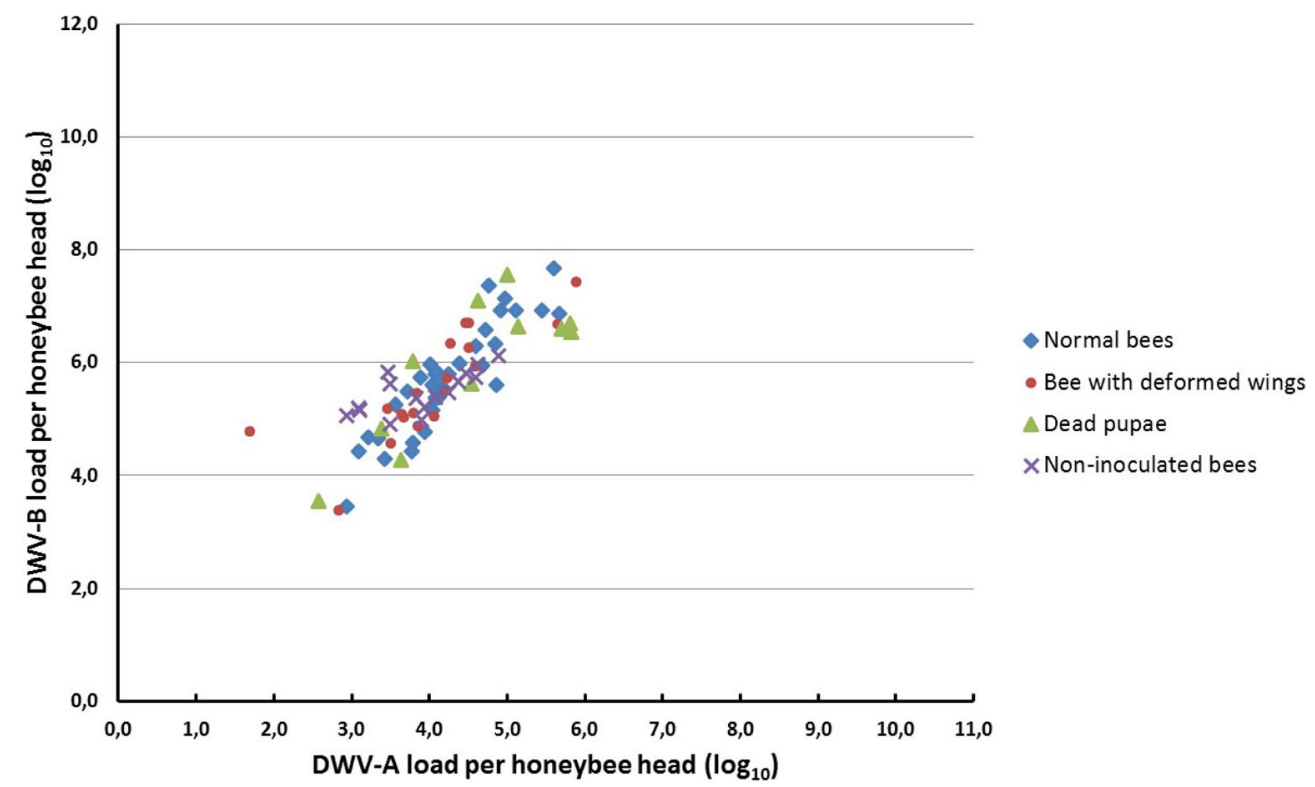

Figure 3. DWV-A and DWV-B loads (in $\log _{10}$ ) quantified by quantitative RT-PCR in control bees. Crosses: noninoculated bees. Control pupae inoculated with phosphate buffer emerging with normal wings (blue diamonds), with wing deformities (red dots), or death during pupation (green triangles). 
Table III. Comparison of DWV variant loads between non-inoculated bees and phosphate buffer (PB) inoculated bees, and between normal-wing bees, bees with wing deformities, and dead pupae in the PB-inoculated control group. The $p$ values were calculated by Student's $t$ test

\begin{tabular}{llll}
\hline Groups & Non-inoculated bees & $\begin{array}{l}\text { PB-bee with wing } \\
\text { deformities }\end{array}$ & PB-dead pupae \\
\hline PB-inoculated bees & DWV-A: $p=0.15 ;$ & $\mathrm{nc}$ & $\mathrm{nc}$ \\
DW-normal bees & $\mathrm{nc}$ & DWV-A: $p=0.35$ & DWV-B: $p=0.36 ;$ \\
$\begin{array}{l}\text { PB-bee with wing } \\
\text { deformities }\end{array}$ & $\mathrm{nc}$ & $\mathrm{nc}$ & DWV-A: $p=0.28 ;$ \\
& & & DWV-B: $p=0.53$ \\
\hline
\end{tabular}

$n c$ not calculated

DWV-A and DWV-B were significantly different from the DWV variant inoculated bees and the corresponding PB control bees $(p<0.01)$.

Taking into account all the honeybees inoculated with DWV variants (shown in Figure 5), the mean of DWV-A viral loads was $7.9 \times 10^{7}$ copies/head, and the DWV-B load mean was 1.7 $\times 10^{9}$ copies/head (Figure 6). These loads were also higher than the viral loads quantified in PB control bees $(p<0.01)$. The mean loads in PB control bees were $1.7 \times 10^{4}$ copies/head for DWVA, and $5.1 \times 10^{5}$ copies/head for DWV-B.

Moreover, dead pupae inoculated with $\mathrm{PB}$, or viral inocula were tested by RT-qPCR amplifying the ABPV, BQCV, CBPV, and SBV (Table V). ABPV was not detected in any inoculated pupae. BQCV was detected in one PB-inoculated pupa at $9.0 \times 10^{2}$ copies/head. CBPV and SBV were quantified in PB-inoculated pupa at a mean load of $3.2 \times 10^{3}$ and $4.9 \times 10^{5}$ copies/head respectively. Among the 15 dead pupae inoculated with viral suspensions, 1 was infected by BQCV at $2.6 \times 10^{2}$ copies/head (pupa inoculated with DWV-B inoculum 2). CBPV was detected in the three dead pupae inoculated with DWV-A inoculum 2 at a mean load of $8.4 \times 10^{4}$ copies/head. This virus was also detected in one pupa inoculated with DWV-B inoculum 2 at CBPV loads of $1.4 \times 10^{3}$ copies/head respectively. SBV was quantified in dead pupae at various load levels. In pupae that had been inoculated with either DWV-B inoculum 1 or DWV-A inoculum 2, the mean SBV load was $9.4 \times 10^{3}$ copies/head. The mean SBV load for dead pupae inoculated with either DWV-A inocula 1 and 3 or DWV-B inoculum 2 was 1.9 $\times 10^{12}$ copies/head.

\subsection{Outcomes and viral loads in the heads of honeybees inoculated with serial dilutions of inocula DWV-A inoculum 2 and DWV-B inoculum 1}

In order to explain the surprising results from Figure 5, we initiated new experimental inoculations of pupae with DWV-A inoculum 2 and DWV-B inoculum 1 adjusted to lower doses (Figure 7). The pupae inoculated with DWV-A inoculum 2 (100 copies of DWV-A) exhibited a significant increase of $3.8 \log _{10}$ copies in average DWV-A load $(p<0.01)$, while the average DWVB load slightly decreased by $0.8 \log _{10}$ copies as compared to those exhibited by the PB control bees $(p=0.01)$. The opposite was observed for pupae inoculated with the diluted DWV-B inoculum 1 inoculum (30 copies of DWV-B) (Figure 7a). A significant increase by $3.2 \log _{10}$ copies in average DWV-B load $(p<0.01)$ was exhibited by the inoculated honeybees while the average DWV-A load was slightly increased by $1.5 \log _{10}$ as compared to the average DWV-A load exhibited by the PB control bees $(p<0.01)$. At 10-fold higher doses, both DWV-A and DWV-B replicated in inoculated pupae (Figure $7 b$ ).

The pupae's outcomes differed according to the inocula used (Figure 8). No increase in pupae mortality was observed notably with both DWVA inoculum $2(p=0.85)$ and DWV-B inoculum 1 $(p=0.70)$ and diluted inocula (containing 100 
copies of DWV-A and 30 copies of DWV-B respectively) compared to $\mathrm{PB}$ control bees (Figure 8a). However, pupae inoculated with DWV-A inoculum 2 containing 100 copies of DWV-A per $2 \mu$ exhibited a high rate of deformed wings $(55-95 \%$ CI [39-71]; $p<0.01)$ while the rate for pupae inoculated with the DWV-B inoculum 1 containing 30 copies of DWV-B per $2 \mu \mathrm{l}$ was similar (24-95\%CI [10-39]; $p=0.91)$ to $\mathrm{PB}$ control bees (23-95\%CI [10-36]) (Figure 8b). A rate of $48 \%$ was found for wing deformities with inoculum DWV-B inoculum 1 containing 300 copies of DWV-B per $2 \mu \mathrm{l}$.

\section{DISCUSSION}

In this study, we analyzed the impact of the inoculation of DWV-A or DWV-B to pupae on three virulence features (i.) rate of pupae mortality, (ii.) rate of bees emerging with wing deformities, and (iii.) viral loads of DWV-A and DWV-B in honeybee heads (amplification targeting either the DWV-A or the DWV-B VP3 coding sequences; (Schurr et al. 2019)). The differences found in the mortality rates could not be correlated with the DWV variant genotype inoculated at $10^{4}$ copies to pupae (Figure 4). We excluded the hypothesis that the deaths were associated with the replication of ABPV, BQCV, or CBPV since these viruses were not frequently detected in dead pupae by RT-qPCR and when they were, their viral load levels (Table V) were below the thresholds previously suggested for overt infections $\left(10^{5}\right.$, $10^{8}$, and $10^{8}$ respectively)(Schurr et al. 2019). However, high viral loads of SBV (above $10^{9}$ copies/head) were detected in the dead pupae inoculated with DWV-A inocula 1 and 3, or DWV-B inoculum 2, but not in those inoculated with PB, DWV-A inoculum 2, or DWV-B inoculum 1 (Table V). Therefore, our results do not indicate that the DWV variant alone can induce mortality at the pupal stage. Mockel et al. (Mockel et al. 2011) reported that DWV did not induce pupal mortality and they suggested that DWV allows infected pupae to develop until emergence. Moreover, two recent studies of DWV virulence on honeybee pupae support this conclusion (Remnant et al. 2019; Tehel et al. 2019). Remnant et al. observed high mortality in 

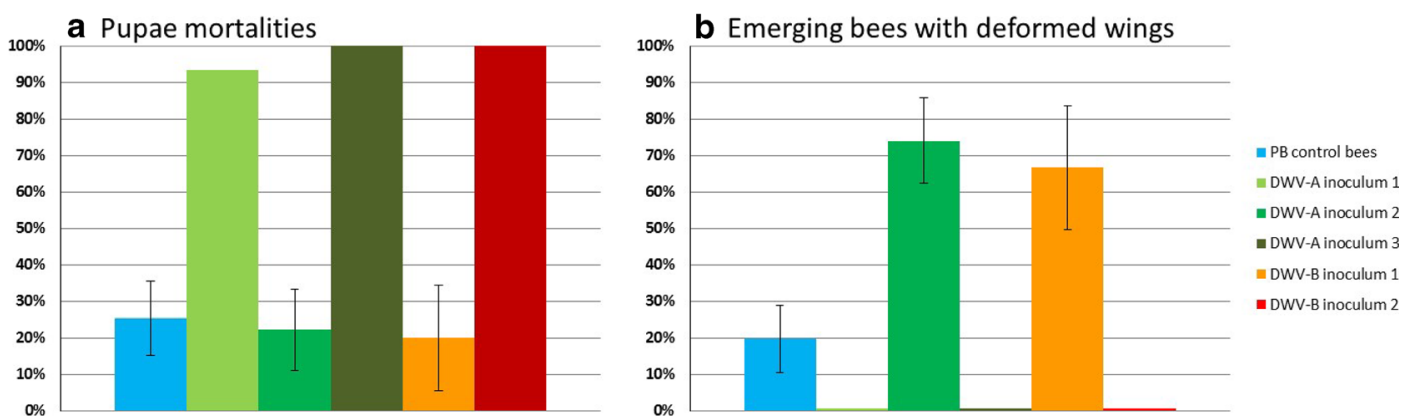

Figure 4. Percentage of pupae mortalities (a) and of emerging bees with deformed wings (b) after inoculation of viral suspensions $\left(10^{4}\right.$ equivalent genome copies per $\left.2 \mu \mathrm{l}\right)$ prepared from the head of naturally infected honeybees in phosphate buffer (PB). PB control bees: 4 replicates for 71 pupae. Honeybees inoculated with viral suspensions containing DWV-A: DWV-A inoculum 1 (1 replicate for 15 pupae), DWV-A inoculum 2 ( 3 replicates for 54 pupae), and DWV-A inoculum 3 (1 replicate for 11 pupae). Honeybees inoculated with viral suspensions containing DWVB: DWV-B inoculum 1 ( 2 replicates for 30 pupae) and DWV-B inoculum 2 (2 replicates for 43 pupae). The bars indicate the $95 \%$ confidence interval of the calculated rates

honeybee pupae when they were inoculated with bee extract containing high levels of SBV and BQCV, whereas pupae inoculated with high levels of DWV resulted in near $100 \%$ survival (Remnant et al. 2019). Tehel et al. found that both DWV-A and DWV-B caused slightly elevated pupal mortality (the mean mortality being $18 \%$ higher than for the controls) (Tehel et al. 2019). Both studies support previous findings suggesting that the $V$. destructor mite could affect negatively the prevalence of highly virulent viruses (Martin et al. 2012; Mondet et al. 2014). Indeed, the virulent viruses (i.e., ABPV or KBV) impair the mite's reproduction by killing the pupae, while viruses with a lower virulence in pupae, enable the mite to complete a full reproduction cycle and subsequently to transmit these less virulent viruses (i.e., DWV variants). However, DWV-induced pupae mortality has been described in two studies (Gisder et al. 2018; Lamp et al. 2016). Despite the fact that the authors did not detect other viruses in the colonies or in the inocula used in their studies, they did not report whether they found other honeybee viruses, such as SBV, in the pupae that died during the experiment.

The rates of bees emerging with deformed wings increased when using inocula where either DWV-A or DWV-B was detected (Figure 4b). Moreover, since both DWV-A and DWV-B variants actively replicated in the heads of bees with deformed wings when injected at $10^{4}$ copies/pupa (Figure 5), we cannot establish whether one genotype was more closely correlated to the emergence of bees with wing deformities. Nevertheless, when we inoculated less concentrated DWV variants, we found a higher rate of bees emerging with deformed wings, DWV-A being associated with an increase in DWV-A load only (Figures $7 \mathrm{a}$ and $8 \mathrm{~b}$ ). However, further experiments with additional strains would be necessary to support this observation. Mockel et al. previously studied the relationship between the quantity of injected DWV and the proportion of bees with wing deformities (Mockel et al. 2011). They reported that an injection of $10^{4} \mathrm{DWV}$ equivalent genome induces deformed wings in about $50 \%$ of emerging bees. We found that this dose led to higher rates (67 to $74 \%$ ) and was comparable to the $60 \%$ elevation in wing deformities reported by Tehel et al. (Tehel et al. 2019). This difference may be due to biological reasons such as the DWV strain used or to differences in the honeybee's tolerance to the infection.

Our results also show that under experimental conditions, wing deformities could not systematically be correlated with an increase in DWV variant loads (Figures 2 and 3). Indeed, no virus was injected into the PB control bees, and the $V$. destructor mite was not detected in either the winter apiary or the brood cells from which the 

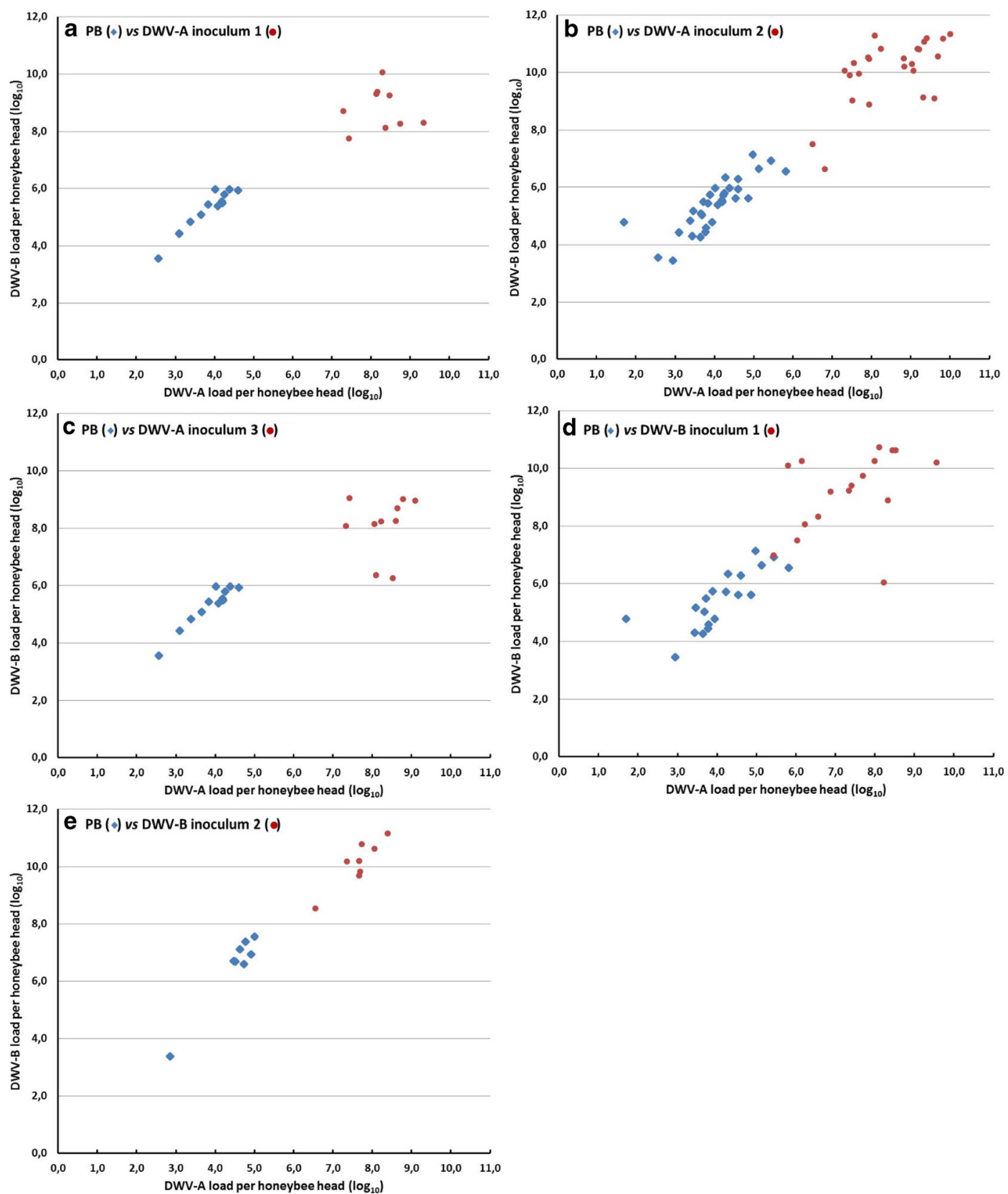

Figure 5. DWV-A and DWV-B loads (in $\log _{10}$ ) quantified in honeybees inoculated with DWV variants $\left(10^{4}\right.$ copies/2 $\mu \mathrm{l}$ ) or in control bees inoculated with phosphate buffer (PB). Blue diamonds: PB control bees; red dots: DWV variant inoculated bees. a-f DWV variant quantification by RT-qPCR in honeybees inoculated with DWV-A inocula 1 to 3, and DWV-B inocula 1 and 2 respectively, and in the corresponding PB control bees.

pupae were harvested. We therefore expected to obtain only living bees with normal wings and low viral loads. Surprisingly, wing deformities were observed in a few of the control bees with moderate viral loads $\left(<10^{7}\right.$ copies/head) similar to the loads of bees emerging with normal wings. 


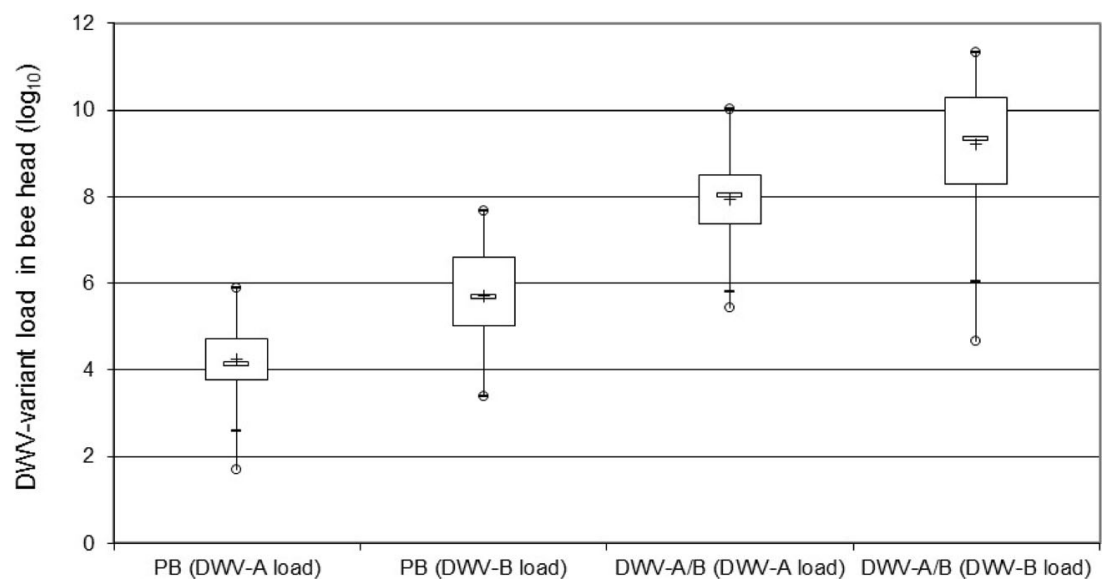

Figure 6. DWV-A or DWV-B loads (in $\log _{10}$ ) quantified in control bees inoculated with phosphate buffer (PB; $n=$ 64 ) or in honeybees inoculated with a DWV variant (DWV-A/B; $n=86$ ). The box plots show the distribution of populations, with the first quartile (25\%) and third quartile (75\%) (box), the median (50\%, white dash), the mean (cross), the minimal and maximal values (whiskers), and outliers (circle).

The stress generated by the inoculation of pupae could explain this unexpected outcome because we found no emerging honeybees with deformed wings in the control group of non-inoculated pupae, although they were quantified with equivalent viral loads (Figure 3). Our observation supports a previous study reporting bees with deformed wings in colonies from $V$. destructor-free areas (Forsgren et al. 2012), even though the suggested source of stress (subnormal temperatures or genetic predispositions) differed from a needle injection. Tehel et al. who experimented with DWV inoculation of pupae, also found control bees with wing deformities despite no significant increase in DWV loads (Tehel et al. 2019). Moreover, we found that DWV-A and DWV-B were circulating in our winter apiary colonies despite no mites being detected (Table II). The capacity of DWV variants to be transmitted horizontally as well as by trophallaxis or vertical transmission (contamination of the eggs of a chronically-infected queen) would explain this background viral circulation within our mite-free hives (Chen et al. 2006; Locke et al. 2017; Mondet et al. 2014; Yue and Genersch, 2005).

The only characteristic clearly distinguishing the DWV variants was the higher viral load of DWV-B than DWV-A in the heads of inoculated bees
(Figure 6). This supports previous conclusions that DWV-B is better able to replicate in honeybees at higher levels through the mite transmission route (Tehel et al. 2019). Nevertheless, our results suggested that there is no correlation between the efficiency of viral replication (Figure 7a) and the capability of DWV-A to efficiently trigger wing deformities in inoculated pupae (Figure 8b). However, these differences in the outcomes of inoculated pupae should be confirmed by extended studies with additional serial dilutions of inoculated DWV variants (at doses between 10 and 1000 copies of DWV variant per pupa). Moreover, other effects have been reported on DWV-infected adult bees, such as learning deficit or premature death (Gisder et al. 2018; Iqbal and Mueller, 2007; McMahon et al. 2016). Studies may be performed to evaluate whether DWV-B has a greater impact on adult bees than on pupae development.

The inoculations of viral suspensions containing either DWV-A or DWV-B at equivalent doses $\left(10^{4}\right.$ copies $\left./ 2 \mu \mathrm{l}\right)$ clearly led to an increased load for both DWV-A and DWV-B (Figure 5). This increase in both DWV variant loads was unexpected. After administering a DWV variant, we expected to measure an increase in viral load of only this particular variant, as reported for 
E. Dubois et al.

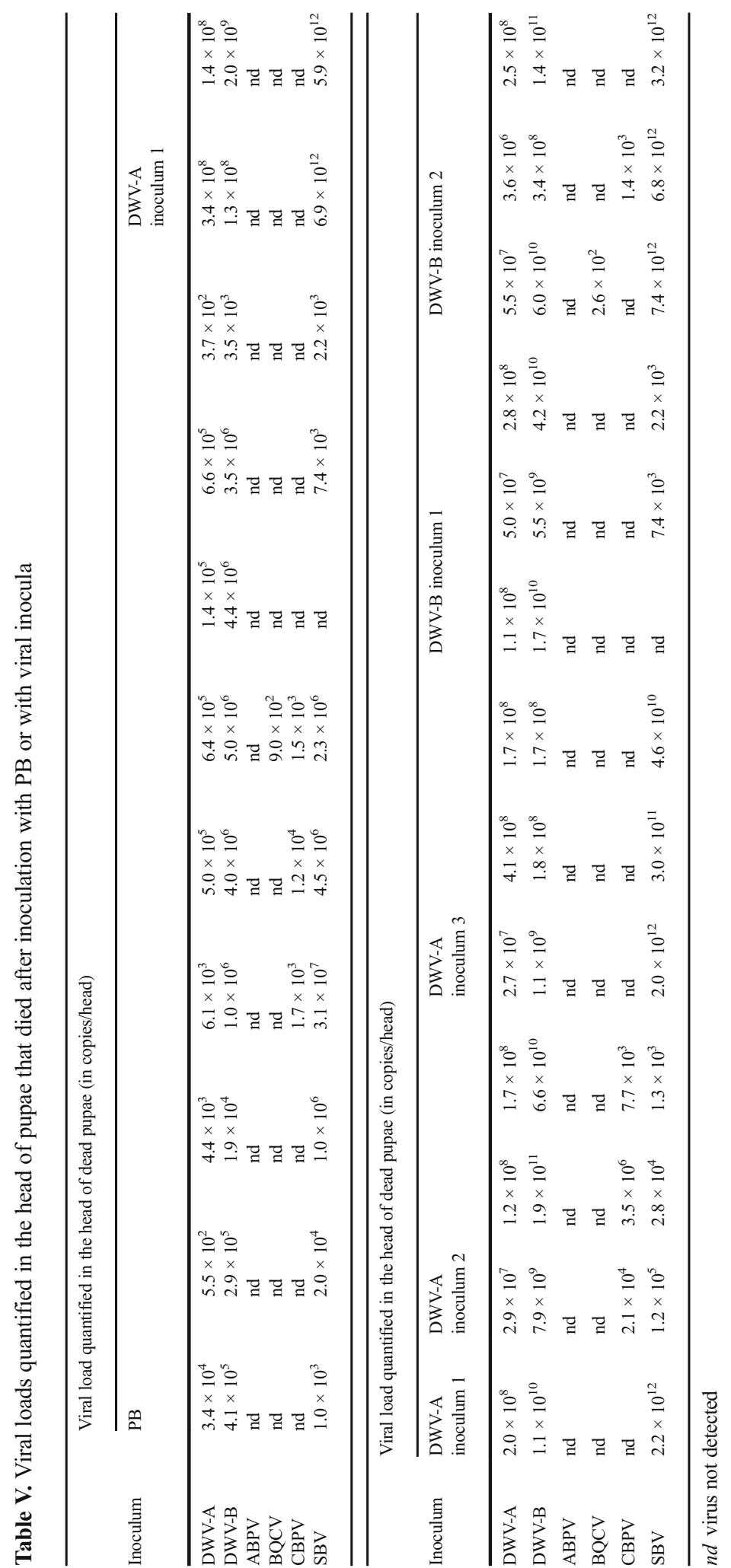



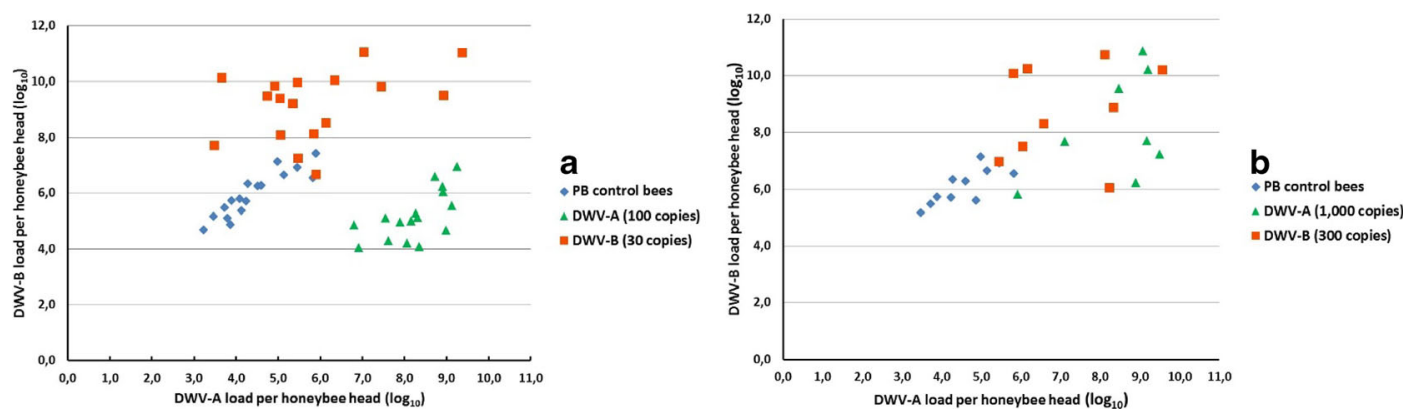

Figure 7. DWV-A and DWV-B loads (in $\log _{10}$ ) quantified in honeybees inoculated with diluted DWV-A inoculum 2 or DWV-B inoculum 1 or in control bees inoculated with phosphate buffer (PB). a Two trials; DWV-A inoculum 2 (100 copies per $2 \mu \mathrm{l}$ ); DWV-B inoculum 1 (30 copies per $2 \mu \mathrm{l}$ ). b One trial; DWV-A inoculum 2 (1000 copies per 2 $\mu \mathrm{l})$; DWV-B inoculum 1 (300 copies per $2 \mu \mathrm{l}$ ). Blue diamonds: PB control bees; green triangles: DWV-A inoculum 2 (DWV-A) inoculated bees; red squares: DWV-B inoculum 1 (DWV-B) inoculated bees

previous experiments (Tehel et al. 2019). The additional DWV variant that replicated in inoculated pupae could either be due to undetected viruses in the inocula or viruses already present in the pupae that were then stimulated by the inocula. Knowing the limits of detection (LOD of DWV-A RT-qPCR $=2.5 \times 10^{2}$ copies $/$ bee and LOD of DWV-B RT-qPCR $=1.0 \times 10^{5}$ copies/bee; (Schurr et al. 2019) and taking into account the dilution factors used to prepared the inoculum (Table IV); we considered unlikely that the minor DWV variant, undetected by RT-qPCR, have impacted our experiments. However, our results suggest that the replication of a DWV variant already infecting the pupa was stimulated by the active replication of the inoculated virus. Zioni et al. suggested a possible "helper" effect for the transmission of DWV by VDV-1 that could also promote recombination between both DWV variants (Zioni et al. 2011). Ryabov et al., who also observed a positive correlation between DWV-A and DWV-B in co-infected colonies, suggested that DWV-A replication could be required for DWV-B to achieve high viral levels (Ryabov et al. 2017). Nevertheless, such "helper" effect seemed to be viral load-dependent and
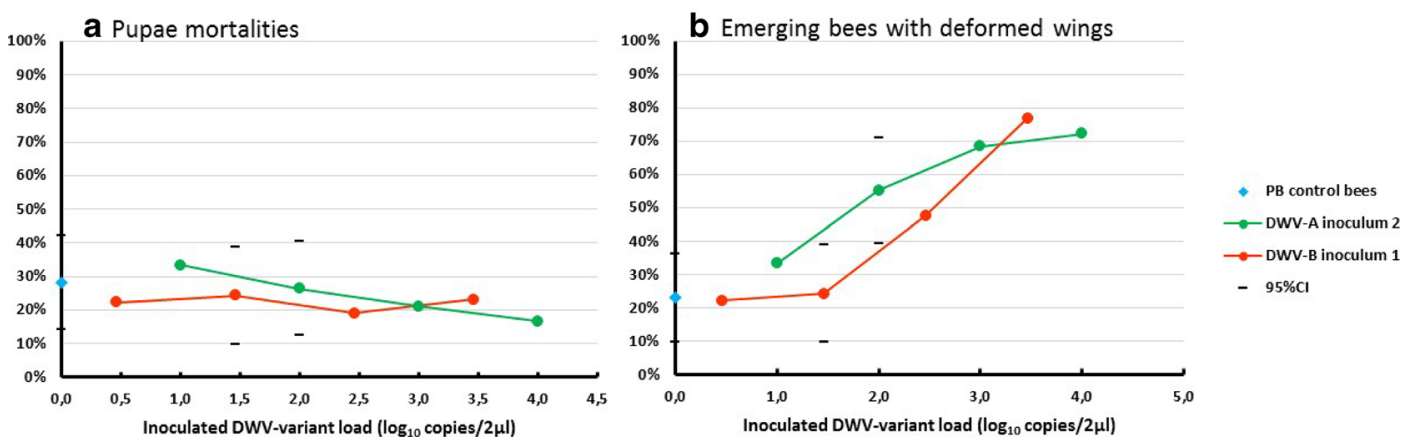

Figure 8. Percentage of pupae mortalities (a) and of emerging bees with deformed wings (B) after inoculation with serial dilutions of DWV-A inoculum 2 or DWV-B inoculum 1. PB: 39 pupae (2 trials) were inoculated with phosphate buffer (control bees). Pupae were inoculated with serial dilutions of DWV-A inoculum 2 containing 10, 100,1000 , and 10,000 copies of DWV-A per $2 \mu \mathrm{l}(n=18,38$ [2 trials], 19, and 18 pupae respectively) or with serial dilutions of DWV-B inoculum 1 containing 3, 30, 300, and 3000 copies of DWV-B per $2 \mu \mathrm{l}(n=18,33$ [2 trials], 21, and 13 pupae respectively). The slots indicate the $95 \%$ confidence interval $(95 \% \mathrm{CI})$ of the calculated rates for PB control bees and duplicated inoculations (DWV-A inoculum 2 at 100 copies of DWV-A and DWV-B inoculum 1 at 30 copies of DWV-B) 
could be correlated with the active replication of DWV inoculated at high doses. We found that pupae inoculated with 100 copies of DWV-A or 30 copies of DWV-B did not exhibit an increase in both DWV variant loads (Figure 7). These results were similar to those found by Tehel et al. after inoculation of $10^{4}$ DWV-A or DWV-B to pupae (Tehel et al. 2019). Notably, they found by RTqPCR an increase of only one DWV variant. In addition, our results suggest that several but not all inocula containing DWV variants could stimulate viral species belonging to the same genus (Iflavirus) such as Sacbrood virus (Table V). Previous studies investigating the co-infection of pupae with SBV and DWV have suggested that the suppression of melanization by SBV could reduce honeybee survival following a DWV infection (Ryabov et al. 2016). Indeed, the active replication of DWV affects the transcription level of Amell102 gene involves in melanization and encapsulation by altering the NF-KB signaling (Di Prisco et al. 2016; Nazzi et al. 2012). However, if such viral synergy between SBV and DWV does really exist, it may be dependent on the viral strains infecting honeybees, the sequence of viral exposure (i.e., exposure to SBV must come before exposure to a DWV variant), the route of viral transmission (trophallaxis versus injection), and/or the age of honeybees exposed to viruses (larvae versus adults).

In conclusion, our results show that both DWV-A and DWV-B were associated with the emergence of bees with deformed wings. The only difference between both DWV variants was the higher DWV-B loads than DWV-A loads quantified by RT-qPCR in the head of inoculated pupae. The mortality of DWV-inoculated pupae was strictly associated with co-infections by DWV variants and SBV. This suggests the need to analyze known honeybee viruses infecting pupae (at least ABPV, BQCV, and $\mathrm{SBV}$ ) when death is observed at pupation under experimental conditions. Finally, the injection of a low dose of DWV variant was sufficient to trigger a high viral load, suggesting that the mite-dependent transmission route in pupae is extremely efficient for inducing overt DWV infection.

\section{ACKNOWLEDGEMENTS}

We are grateful to Delphine Libby-Claybrough, professional translator and native English speaker, for revising the paper.

\section{Funding information}

This study was supported by the European Commission through the 7th framework collaborative project SMARTBEES (Sustainable Management of Resilient Bee Populations, grant agreement KBBE-7-61393).

Devenir des nymphes d'abeilles inoculées avec les génotypes $A$ et $B$ du virus des ailes déformées.

DWV / Varroa destructor virus-1 / virulence / déformation de l'aile / mortalité des pupes.

Auswirkungen einer Imfpfung von Honigbienenpuppen mit den Genotypen A und B des Deformed Wing Virus.

DWV / Varroa destructor virus-1 / Virulenz / Flügeldeformation /Puppenmortalität.

\section{REFERENCES}

Amiri, E., Strand, M.K., Rueppell, O., Tarpy, D.R., 2017. Queen quality and the impact of honey bee diseases on queen health: potential for interactions between two major threats to colony health. Insects 8 .

Benaets, K., Van Geystelen, A., Cardoen, D., De Smet, L., de Graaf, D.C., Schoofs, L., Larmuseau, M.H., Brettell, L.E., Martin, S.J., Wenseleers, T., 2017. Covert deformed wing virus infections have longterm deleterious effects on honeybee foraging and survival. Proc Biol Sci 284.

Blanchard, P., Guillot, S., Antunez, K., Koglberger, H., Kryger, P., de Miranda, J.R., Franco, S., Chauzat, M.P., Thiery, R., Ribiere, M., 2014. Development and validation of a real-time two-step RT-qPCR $\operatorname{TaqMan}((\mathrm{R}))$ assay for quantitation of Sacbrood virus (SBV) and its application to a field survey of symptomatic honey bee colonies. J Virol Methods 197, 713.

Blanchard, P., Regnault, J., Schurr, F., Dubois, E., Ribiere, M., 2012. Intra-laboratory validation of chronic bee paralysis virus quantitation using an accredited standardised real-time quantitative RT-PCR method. J Virol Methods 180, 26-31. 
Bowen-Walker, P.L., Martin, S.J., Gunn, A., 1999. The transmission of deformed wing virus between honeybees (Apis mellifera L.) by the ectoparasitic mite varroa jacobsoni Oud. J Invertebr Pathol 73, 101-106.

Chantawannakul, P., Ward, L., Boonham, N., Brown, M., 2006. A scientific note on the detection of honeybee viruses using real-time PCR (TaqMan) in Varroa mites collected from a Thai honeybee (Apis mellifera) apiary. J Invertebr Pathol 91, 69-73.

Chen, Y., Evans, J., Feldlaufer, M., 2006. Horizontal and vertical transmission of viruses in the honey bee, Apis mellifera. Journal of Invertebrate Pathology 92, 152-159.

Cornman, R.S., 2017. Relative abundance of deformed wing virus, Varroa destructor virus 1, and their recombinants in honey bees (Apis mellifera) assessed by kmer analysis of public RNA-Seq data. J Invertebr Pathol 149, 44-50.

Crailsheim, K., Brodschneider, R., Aupinel, P., Behrens, D., Genersch, E., Vollmann, J., Riessberger-Gallé, U., 2013 Standard methods for artificial rearing of Apis mellifera larvae. In V Dietemann; J D Ellis; P Neumann (Eds) The COLOSS BEEBOOK, Volume I: standard methods for Apis mellifera research. Journal of Apicultural Research 52.

Dainat, B., Evans, J.D., Chen, Y.P., Gauthier, L., Neumann, P., 2012a. Dead or alive: deformed wing virus and Varroa destructor reduce the life span of winter honeybees. Appl Environ Microbiol 78, 981-987.

Dainat, B., Evans, J.D., Chen, Y.P., Gauthier, L., Neumann, P., 2012b. Predictive markers of honey bee colony collapse. PLoS One 7, e32151.

Dalmon, A., Desbiez, C., Coulon, M., Thomasson, M., Le Conte, Y., Alaux, C., Vallon, J., Moury, B., 2017. Evidence for positive selection and recombination hotspots in Deformed wing virus (DWV). Sci Rep 7, 41045.

de Miranda, J.R., Cordoni, G., Budge, G., 2010. The acute bee paralysis virus-Kashmir bee virus-Israeli acute paralysis virus complex. J Invertebr Pathol 103 Suppl 1, S30-47.

DeGrandi-Hoffman, G., Chen, Y., Simonds, R., 2013. The effects of pesticides on queen rearing and virus titers in honey bees (Apis mellifera L.). Insects 4, 71-89.

Di Prisco, G., Annoscia, D., Margiotta, M., Ferrara, R., Varricchio, P., Zanni, V., Caprio, E., Nazzi, F., Pennacchio, F., 2016. A mutualistic symbiosis between a parasitic mite and a pathogenic virus undermines honey bee immunity and health. Proc Natl Acad Sci U S A 113, 3203-3208.

Di Prisco, G., Cavaliere, V., Annoscia, D., Varricchio, P., Caprio, E., Nazzi, F., Gargiulo, G., Pennacchio, F., 2013. Neonicotinoid clothianidin adversely affects insect immunity and promotes replication of a viral pathogen in honey bees. Proc Natl Acad Sci U S A $110,18466-18471$.

Di Prisco, G.D., Zhang, X., Pennacchio, F., Caprio, E., Li, J., Evans, J.D., Degrandi-Hoffman, G., Hamilton, M., Chen, Y.P., 2011. Dynamics of persistent and acute deformed wing virus infections in honey bees, Apis mellifera. Viruses 3, 2425-2441.
Dietemann, V., Nazzi, F., Martin, S.J., Anderson, D.L., Locke, B., Delaplane, K.S., Wauquiez, Q., Tannahill, C., Frey, E., Ziegelmann, B., Rosenkranz, P., Ellis, J.D., 2013 Standard methods for varroa research. In V Dietemann; J D Ellis; P Neumann (Eds) The COLOSS BEEBOOK, Volume II: standard methods for Apis mellifera pest and pathogen research. Journal of Apicultural Research 52.

Forsgren, E., Fries, I., de Miranda, J.R., 2012. Adult honey bees (Apis mellifera) with deformed wings discovered in confirmed varroa-free colonies. Journal of Apicultural Research 51, 136-138.

Francis, R.M., Nielsen, S.L., Kryger, P., 2013. Varroa-virus interaction in collapsing honey bee colonies. PLoS One 8, e57540.

Fujiyuki, T., Takeuchi, H., Ono, M., Ohka, S., Sasaki, T., Nomoto, A., Kubo, T., 2004. Novel insect picorna-like virus identified in the brains of aggressive worker honeybees. J Virol 78, 1093-1100.

Genersch, E., von der Ohe, W., Kaatz, H., Schroeder, A., Otten, C., Büchler, R., Berg, S., Ritter, W., M"uhlen, W., Gisder, S., Meixner, M., Liebig, G., Rosenkranz, P., 2010. The German bee monitoring project: a long term study to understand periodically high winter losses of honey bee colonies. Apidologie 41, 332-352.

Gisder, S., Aumeier, P., Genersch, E., 2009. Deformed wing virus: replication and viral load in mites (Varroa destructor). J Gen Virol 90, 463-467.

Gisder, S., Mockel, N., Eisenhardt, D., Genersch, E., 2018. In vivo evolution of viral virulence: switching of deformed wing virus between hosts results in virulence changes and sequence shifts. Environ Microbiol 20, 4612-4628.

Greiner, M., Gardner, I.A., 2000. Application of diagnostic tests in veterinary epidemiologic studies. Prev Vet Med 45, 43-59.

Iqbal, J., Mueller, U., 2007. Virus infection causes specific learning deficits in honeybee foragers. Proc Biol Sci. 274, 1517-1521.

Jamnikar Ciglenecki, U., Toplak, I., 2012. Development of a real-time RT-PCR assay with TaqMan probe for specific detection of acute bee paralysis virus. J Virol Methods 184, 63-68.

Kevill, J.L., Highfield, A., Mordecai, G.J., Martin, S.J., Schroeder, D.C., 2017. ABC assay: method development and application to quantify the role of three DWV master variants in overwinter colony losses of European honey bees. Viruses 9.

Lamp, B., Url, A., Seitz, K., Eichhorn, J., Riedel, C., Sinn, L.J., Indik, S., Koglberger, H., Rumenapf, T., 2016. Construction and rescue of a molecular clone of deformed wing virus (DWV). PLoS One 11, e0164639.

Lanzi, G., de Miranda, J.R., Boniotti, M.B., Cameron, C.E., Lavazza, A., Capucci, L., Camazine, S.M., Rossi, C., 2006. Molecular and biological characterization of deformed wing virus of honeybees (Apis mellifera L.). J Virol 80, 4998-5009.

Locke, B., Forsgren, E., de Miranda, J.R., 2014. Increased tolerance and resistance to virus infections: a possible 
factor in the survival of Varroa destructor-resistant honey bees (Apis mellifera). PLoS One 9, e99998.

Locke, B., Semberg, E., Forsgren, E., de Miranda, J.R., 2017. Persistence of subclinical deformed wing virus infections in honeybees following Varroa mite removal and a bee population turnover. PLoS One 12, e0180910.

Martin, S.J., Highfield, A.C., Brettell, L., Villalobos, E.M., Budge, G.E., Powell, M., Nikaido, S., Schroeder, D.C., 2012. Global honey bee viral landscape altered by a parasitic mite. Science 336, 1304-1306.

McMahon, D.P., Natsopoulou, M.E., Doublet, V., Furst, M., Weging, S., Brown, M.J., Gogol-Doring, A., Paxton, R.J., 2016. Elevated virulence of an emerging viral genotype as a driver of honeybee loss. Proc Biol Sci 283

Mockel, N., Gisder, S., Genersch, E., 2011. Horizontal transmission of deformed wing virus: pathological consequences in adult bees (Apis mellifera) depend on the transmission route. J Gen Virol 92, 370-377.

Mondet, F., de Miranda, J.R., Kretzschmar, A., Le Conte, Y., Mercer, A.R., 2014 On the front line: quantitative virus dynamics in honeybee (Apis mellifera L.) colonies along a new expansion front of the parasite Varroa destructor. PLoS Pathog 10, e1004323.

Moore, J., Jironkin, A., Chandler, D., Burroughs, N., Evans, D.J., Ryabov, E.V., 2011. Recombinants between Deformed wing virus and Varroa destructor virus-1 may prevail in Varroa destructor-infested honeybee colonies. J Gen Virol 92, 156-161.

Mordecai, G.J., Brettell, L.E., Martin, S.J., Dixon, D., Jones, I.M., Schroeder, D.C., 2016a. Superinfection exclusion and the long-term survival of honey bees in Varroa-infested colonies. ISME J 10, 1182-1191.

Mordecai, G.J., Wilfert, L., Martin, S.J., Jones, I.M., Schroeder, D.C., 2016b. Diversity in a honey bee pathogen: first report of a third master variant of the Deformed Wing Virus quasispecies. ISME J 10, 1264-1273.

Nazzi, F., Brown, S.P., Annoscia, D., Del Piccolo, F., Di Prisco, G., Varricchio, P., Della Vedova, G., Cattonaro, F., Caprio, E., Pennacchio, F., 2012. Synergistic parasite-pathogen interactions mediated by host immunity can drive the collapse of honeybee colonies. PLoS Pathog 8, e1002735.

Ongus, J.R., Peters, D., Bonmatin, J.M., Bengsch, E., Vlak, J.M., van Oers, M.M., 2004. Complete sequence of a picorna-like virus of the genus Iflavirus replicating in the mite Varroa destructor. J Gen Virol 85, 3747-3755.

Remnant, E.J., Mather, N., Gillard, T.L., Yagound, B., Beekman, M., 2019. Direct transmission by injection affects competition among RNA viruses in honeybees. Proc. R. Soc. B 286:, 20182452.

Ryabov, E.V., Childers, A.K., Chen, Y., Madella, S., Nessa, A., vanEngelsdorp, D., Evans, J.D., 2017. Recent spread of Varroa destructor virus-1, a honey bee pathogen, in the United States. Sci Rep 7, 17447.

Ryabov, E.V., Fannon, J.M., Moore, J.D., Wood, G.R., Evans, D.J., 2016. The Iflaviruses Sacbrood virus and Deformed wing virus evoke different transcriptional responses in the honeybee which may facilitate their horizontal or vertical transmission. PeerJ 4, e1591.

Ryabov, E.V., Wood, G.R., Fannon, J.M., Moore, J.D., Bull, J.C., Chandler, D., Mead, A., Burroughs, N., Evans, D.J., 2014. A virulent strain of deformed wing virus (DWV) of honeybees (Apis mellifera) prevails after Varroa destructor-mediated, or in vitro, transmission. PLoS Pathog 10, e1004230.

Schurr, F., Cougoule, N., Riviere, M.P., Ribiere-Chabert, M., Achour, H., Adam, D., Castillo, C., de Graaf, D.C., Forsgren, E., Granato, A., Heinikainen, S., Jurovcikova, J., Kryger, P., Manson, C., Menard, M.F., Perennes, S., Schafer, M.O., Ibanez, E.S.M., Silva, J., Gajger, I.T., Tomkies, V., Toplak, I., Viry, A., Zdanska, D., Dubois, E., 2017. Trueness and precision of the real-time RT-PCR method for quantifying the chronic bee paralysis virus genome in bee homogenates evaluated by a comparative inter-laboratory study. J Virol Methods 248, 217-225.

Schurr, F., Tison, A., Militano, L., Cheviron, N., Sircoulomb, F., Riviere, M.P., Ribiere-Chabert, M., Thiery, R., Dubois, E., 2019. Validation of quantitative real-time RT-PCR assays for the detection of six honeybee viruses. J Virol Methods 270, 70-78.

Shah, K.S., Evans, E.C., Pizzorno, M.C., 2009. Localization of deformed wing virus (DWV) in the brains of the honeybee, Apis mellifera Linnaeus. Virol J 6, 182.

Strauss, U., Human, H., Gauthier, L., Crewe, R.M., Dietemann, V., Pirk, C.W., 2013. Seasonal prevalence of pathogens and parasites in the savannah honeybee (Apis mellifera scutellata). J Invertebr Pathol 114, 45-52.

Tehel, A., Vu, Q., Bigot, D., Gogol-Döring, A., Koch, P., Jenkins, C., Doublet, V., Theodorou, P., Paxton, R., 2019. The two prevalent genotypes of an emerging infectious disease, Deformed wing virus, cause equally low pupal mortality and equally high wing deformities in host honey bees. Viruses 11, 114.

Wells, T., Wolf, S., Nicholls, E., Groll, H., Lim, K.S., Clark, S.J., Swain, J., Osborne, J.L., Haughton, A.J., 2016. Flight performance of actively foraging honey bees is reduced by a common pathogen. Environ Microbiol Rep 8, 728-737.

Yue, C., Genersch, E., 2005. RT-PCR analysis of deformed wing virus in honeybees (Apis mellifera) and mites (Varroa destructor). J Gen Virol 86, 3419-3424.

Yue, C., Schroder, M., Gisder, S., Genersch, E., 2007. Vertical-transmission routes for deformed wing virus of honeybees (Apis mellifera). J Gen Virol 88, 23292336.

Zioni, N., Soroker, V., Chejanovsky, N., 2011. Replication of Varroa destructor virus 1 (VDV-1) and a Varroa destructor virus 1-deformed wing virus recombinant (VDV-1-DWV) in the head of the honey bee. Virology 417, 106-112.

Publisher's note Springer Nature remains neutral with regard to jurisdictional claims in published maps and institutional affiliations. 